\title{
Neonatal Rickets
}

National Cancer Institute

\section{Source}

National Cancer Institute. Neonatal Rickets. NCI Thesaurus. Code C99233.

Rickets that usually affects premature infants. Factors that contribute to the development of neonatal rickets include calcium, phosphorus, or calciferol deficiency. It manifests with spontaneous fractures, respiratory distress, and growth developmental delays. 